\title{
Multimedia y nuevas tendencias tecnológicas y comunicativas en documentación informativa a propósito de Bibliored 3.0
}

\section{Servicio Documentación Multimedia}

\section{Universidad Complutense de Madrid}

\section{Recibido: 20 de septiembre de 2015}

\section{Aceptado: 12 de octubre de 2015}

\section{Resumen}

Se traza una breve referencia sobre el Servicio de Documentación Multimedia de la Universidad Complutense y sus actuaciones en el ámbito de la documentación informativa durante sus 23 años de historia (1993-2015), para describir a continuación la estructura de contenidos y aportaciones del proyecto más reciente del servicio, Bibliored 3.0. Para centrarse y desarrollar más ampliamente las nuevas tendencias tecnológicas que afectan a la documentación informativa y al proceso documental en medios de comunicación. Se destaca finalmente la producción informativa que se está llevando a cabo desde Bibliored para la difusión de dichas nuevas tendencias.

\section{Pallabras Clave}

Bibliored3.0, competencia informacional, multimedia, multiplataforma, transmedia, métricas web social

\section{Multimedia and new technological trends in management information mass media about Bibliored 3.0}

\section{Albstract}

A brief reference Multimedia Documentation Service of the Complutense University and its activities in the field of management information mass media during 23 years history (1993-2015). Description the structure and content of the project recent: Bibliored 3.0. New technological trends about management and process information mass media: production and dissemination by Bibliored

\section{Keywords}

Bibliored3.0, information literacy, multi platform, multimedia, transmedia, social web metrics

http://dx.doi.org/10.5209/rev CDMU.2015.v26.50635 


\section{INTRODUCCIÓN}

El Servicio de Documentación Multimedia de la Universidad Complutense de Madrid se crea en 1993 en el marco del Departamento de Biblioteconomía y Documentación de dicha universidad, obteniendo patente número M 2202582 registrada con fecha 20.5.1999 en el Boletín Oficial de la Propiedad Industrial -BOPI, 1.7.1999- (Servicio Documentación Multimedia, 1993-2015).

Desde entonces, de forma ininterrumpida hasta la actualidad ha seguido desarrollando numerosas actividades académicas: proyectos de investigación, publicaciones electrónicas, revistas, portales, canales, blogs, presencia en redes sociales generales y especializadas; con actuaciones en todos estos años en el ámbito de la formación, congresos, asociaciones, producción multimedia propia, difusión informativa, gozando de un buen posicionamiento. Así como sus actuaciones más recientes, 2013-2015 (RTVDoc, Cinedocnet, Redauvi, Youtube, Bibliored), con mención en todo ese tiempo a las relacionadas estrechamente con el entorno latinoamericano, y recientemente y más concretamente con algunas referencias al ámbito brasileño (López, 2007-2013/2015).

Bibliored3.0, como proyecto más reciente (inicia sus primeros pases en octubre de 2014) del Servicio de Documentación Multimedia, viene a integrar y canalizar prácticamente toda su producción de esos 23 años de historia (1993-2015), facilitando asimismo acceso a muy amplia documentación informativa de otros ámbitos especializados.

\section{BIBLIORED3.0: MULTIMEDIA EN BIBLIOTECAS Y SERVICIOS ESPECIALIZADOS UNIVERSITARIOS}

Bibliored3.0 canaliza tanto producción propia como acceso a producción ajena relacionada con los contenidos de dicha web. Su objetivo fundamental por tanto es la producción propia de contenidos multimedia sobre ciencias de la información y comunicación, en bibliotecas y servicios especializados universitarios. Sus contenidos presentan enunciados generales que incluyen sus correspondientes despliegues temáticos en forma de páginas-subpáginas: Qué es-quiénes somos, Bibliotecas, Servicios, Ciencias Información, Formación, Recursos documentales, Redes sociales, Tecnología, Iberoamérica. La producción propiamente dicha de contenidos multimedia y su difusión se lleva a cabo a través del canal RTVDoc desde 2008, realizándose la recuperación de los mismos mediante etiquetas y búsqueda avanzada (los documentos están accesibles a través de cualquier palabra contenida en ellos).

La producción multimedia propia y el acceso a producción ajena relacionada se materializa, por ejemplo, en y con las siguientes herramientas: canales, videotecas fototeca, fonotecas portales comunicación, servicio alertas, agregador multimedia RSS. Y la búsqueda avanzada posibilita el acceso, entre otras muchas, a temáticas como éstas: metodología, web semántica, patrimonio, hispana-europeana, iberoamérica (formación-doctorado-defensa tesis doctorales) y a otros muy variados y pertinentes ámbitos de contenidos relacionados con información y documentación -y su estudio- en bibliotecas y servicios especializados (de información, documentación, investigación) universitarios, y su aplicación a los mismos (Bibliored3.0, 2015a).

Se remite a algunos ejemplos de difusión de Bibliored3.0 a través de blogs, portales y redes sociales como Biblogtecarios espacio web colaborativo sobre información y documentación (Biblogtecarios, 2015) o Biblogsfera (Biblogsfera, 2014). Además de estos ámbitos de difusión deben también destacarse El documentalista audiovisual (EDA, 2015) o Universo Abierto, blog de la biblioteca de la Facultad de 
Documentación y Traducción de la Universidad de Salamanca (Universo Abierto, 2014). O a través de los EPrints Complutense ya mencionados, también accesibles a través de REBIUN y su directorio de repositorios (REBIUN, 2015). A destacar asimismo la difusión informativa de Bibliored 3.0 en ámbitos docentes y de investigación, y foros profesionales como, en octubre de 2014, el VI Congreso Internacional de Bibliotecología, Documentación, Archivística y Museología /Bolivia-La Paz (Centro Cultural España La Paz, 2014). En fin, presencia de las realizaciones del Servicio de Documentación Multimedia en Hispana-Europeana (HispanaEuropeana, 2015; Ramos, Arquero, 2015).

En fin, debe destacarse la edición de una grabación videográfica de 4 minutos 11 segundos de duración, de fecha junio de 2015, accesible a través del canal RTVDoc que mantiene en YouTube el Servicio de Documentación Multimedia. El documento ha sido elaborado por alumnos de la disciplina "Documentación informativa" que se imparte en la Licenciatura de Documentación en la Facultad de Ciencias de la información de la Univesidad Complutense, con guión y supervisión del propio Servicio (Servicio Documentación Multimedia, 2015).

\section{NUEVAS TENDENCIAS TECNOLÓGICAS Y COMUNICATIVAS EN DOCUMENTACIÓN INFORMATIVA Y} EN EL PROCESO DOCUMENTAL

Están proliferando, ininterrumpidamente, nuevas fórmulas y estructuras de la comunicación, de la documentación, de la narración, de las narrativas multimedia, de la multi-interdisciplinariedad, o no tan nuevas pero cuya evolución es continua y cambiante: nuevas narrativas multimedia o transmedia, narrativas transmediales, crossmedia, distribución multiplataforma, multiplicidad autoral, competencias informacionales, transalfabetización, tecnologías creativas, métricas en la web social... nuevas funciones en el ámbito de la documentación informativa (que conllevan nuevas tareas documentales con objeto de interrelacionar fondos documentales y no sólo de establecerlos): convergencia mediática, transversalidad, cultura participativa e inteligencia colectiva, distribución y consumo de cultura, pedagogías críticas, alfabetizaciones y discursos múltiples... Temáticas todas ellas sustentadas en las investigaciones de numerosos especialistas (Cuevas-Cervero, Marques, Sales, 2014; Díaz, Tous, 2012; González, 2015; López, 2015; Miranda, 2015; Peres, Miranda, Simeâo, 2015; Ponjuán, Pinto, Uribe-Tirado; Scolari, 2013, Uribe-Tirado, 2015).

En el sentido apuntado en cuanto a nuevas tendencias concretamente aplicadas al ámbito de estudio y desarrollos de la documentación informativa, resultan muy interesantes e imprescindibles por su actualidad y oportunidad las conclusiones extraídas de la investigación más reciente de los profesores de la Universidades Rey Juan Carlos y Complutense de Madrid, Antonio García Jiménez y Félix del Valle Gastaminza.

"Cambios constantes y profundos, que afectan a los procesos documentales. Proliferación de teléfonos móviles evolucionados (3G y 4G), tabletas, aplicaciones informáticas, definitiva digitalización de los documentos tradicionales, crisis del modelo de negocio de la prensa, mutaciones en las tareas de los periodistas y en su relación con la Documentación..." “...Algunas de esas mutaciones tienen que ver con cambios en los procesos documentales que gestionan el conocimiento registrado: la selección, el tratamiento documental, la recuperación de información y documentos o la preservación documental... (García, Valle, 2015). Añaden estos mismos autores: “... En la selección documental se debe hacer frente a fenómenos como el aumento del flujo de contenidos o la diversificación de los formatos de documentos, con la aparición de nuevas narrativas multimedia o transmedia que definen nuevas tareas documentales: no se trata tanto de 
construir fondos documentales como de interrelacionarlos. En lo que se refiere al tratamiento documental asistimos al cambio de la indización mediante descriptores o palabras clave controladas y definidas a priori por especialistas, sustituida por el etiquetado, una indización libre con un incuestionable componente social". Matizan señalando “... Intervienen también nuevos instrumentos o lenguajes y modelos conceptuales pensados para representar el conocimiento en ámbitos amplios de recuperación que van más allá de las tradicionales normas de tesauros o encabezamientos de materia. En cuanto a la recuperación de información... un buscador, Google, se ha situado en el centro del debate de la recuperación tanto en el ámbito de la discusión periodística como en una visión más sociológica y algunos de los más certeros estudios sobre los usos profesionales y críticos se están haciendo desde nuestra área".

Y también destacan: "En cuanto a la preservación documental... a los cambios en los procesos, las herramientas y las competencias profesionales de la Documentación hay que sumar el acceso generalizado a herramientas y dispositivos tecnológicos. En un contexto en el que se accede a información y documentos de todo tipo a través de tablets y smartphones y donde los medios sociales son el espacio virtual de trabajo y ocio, surgen nuevos retos tanto para la formación como para la praxis profesional. La aparición constante de una gran cantidad de aplicaciones orientadas al usuario parece confirmar la simplicidad, en realidad solo aparente, de los procesos de búsqueda, recuperación y organización de la información. En efecto, existe el riesgo de creer que cualquier cuantificación representa la realidad, que la tecnología es neutral y que los simples datos no contextualizados pueden generar conocimiento. El control del acceso a la memoria e incluso la construcción de la memoria ha sido, es y será un elemento básico para la sociedad... Se hace pues necesario reconsiderar la figura del profesional de la información y la documentación, en un marco donde surgen nuevos perfiles profesionales como los gestores de información, los community managers o los curadores de contenido y en un contexto en el que la Documentación, y otras ciencias humanas y sociales, están en el punto de mira de la sospecha utilitarista y de una reubicación transdisciplinar. Desde nuestro punto de vista, los retos siguen siendo los mismos y, vistas las facilidades para el acceso a una enorme cantidad de datos, se incrementa incluso la necesidad de construir contextos que permitan ofrecer información crítica, útil y fiable".

\section{DIFUSIÓN INFORMATIVA A TRAVÉS DE BIBLIORED 3.0 Y OTROS ÁMBITOS INFORMATIVOS}

Nuevas fórmulas y espacios narrativos, cultura participativa e inteligencia colectiva... tecnologías para la distribución simultánea de información... cultura de la convergencia... redes sociales e internet en producción y difusión cultural... en el ámbito de la documentación informativa o gestión de la información en medios de comunicación. (Bibliored3.0, 2015b). Con acceso a las siguientes manifestaciones:

I Jornadas "Nuevos espacios para la alfabetización e inteligencias múltiples" 7-8 mayo 2015, Universidad de Murcia -Facultad de Matemáticas y Aulario General- sobre las alfabetizaciones necesarias en un mundo caracterizado por la multiplicidad de formatos, de canales de comunicación, de géneros y de discursos. Con temas como la alfabetización transmedia, cine y educación, pedagogías críticas y discursos publicitarios, y alfabetizaciones para el empoderamiento. Un ejemplo de ello es la ponencia de la profesora Mar Grandío en torno al tema "Alfabetización transmedia en los jóvenes universitarios. El transmedia en la universidad" (Grandío, 2015).

"Transmedia y nuevos contenidos digitales": consecuencias que los entornos digitales, representados por las narraciones transmedia están causando en la sociedad. Espacio Fundación Telefónica,15-17 de octubre de 2014. Ciclo Transmedia en Youtube. Narrativa Transmedia (Fundación Telefónica, 2014). 
Icono14. Temática a desarrollar en el número de septiembre de 2015: "Narrativa transmedia: resignificando el consumo mediático", invitando a participar en su elaboración con la siguiente presentación:

"El consumidor se constituye como un nuevo agente productor de las industrias culturales. En plena época de la convergencia mediática y cultural, parece que todos los microrelatos penden de un mismo núcleo generador como es el relato transmedia. Hoy día el consumidor no sólo cambia de canal con el mando, sino que se conecta a la página web de su programa favorito mientras lo emiten en directo, twittea lo que sucede en tiempo real, lee resúmenes del próximo episodio en su móvil o tablet, comenta en foros en Internet, escribe en su blog, linkea contenidos en su página web personal, compra el merchandising oficial e incluso, con algo de pericia e imaginación, puede generar tramas narrativas alternativas y difundirlas por canales no oficiales... “... El giro paulatino en el comportamiento psicosocial de los sujetos individuales y la audiencia, ha provocado que el clásico paradigma comunicacional se haya visto distorsionado en los últimos años por la abrumadora penetración de las TIC y de las herramientas de intercambio entre usuarios que éstas procuran. El espectador no se conforma ya con una única experiencia perceptiva, sino que desea sentirse parte del relato y participar activamente en él. Nos interesa especialmente atender a los nuevos relatos que el transmedia promueve. Desde las nuevas fórmulas narrativas empleadas en los medios de comunicación tradicionales hasta aquellos relatos específicamente producidos para existir en varias tecnologías de distribución simultáneamente, así como el papel de prosumidor que puede adquirir la audiencia en la actualidad" (Icono 14, 2015).

\section{CONCLUSIONES}

Narrativas multimedia, transmedia, narrativas transmediales, crossmedia, distribución multiplataforma, competencias informacionales, tecnologías creativas... como nuevas fórmulas y estructuras de la comunicación, de la documentación, de la narración... que están proliferando más recientemente.

Nuevas tendencias tecnológicas que están afectando al ámbito de la documentación informativa o gestión de la información en y sobre medios de comunicación: nuevas funciones en el ámbito de la documentación informativa que conllevan nuevas tareas documentales con objeto de interrelacionar fondos documentales y no sólo de establecerlos...

Nuevos retos, en consecuencia, para la documentación informativa y los correspondientes procesos documentales: cambios constantes y profundos en las tareas documentales que gestionan el conocimiento registrado.

Cambios trascendentales, en suma, que también afectan al tratamiento documental y toda la cadena documentaal y, como consecuencia, a la preservación documental.

Bibliored3.0, la actuación académica más reciente del Servicio de Documentación Multimedia de la Universidad Complutense, en cuyo ámbito se administra y mantiene, está contribuyendo a la producción y difusión informativa sobre dichas nuevas tendencias.

\section{REFERENCIAS BILIOGRÁFICAS}

Bibliored3.0 (2015a). Página de bienvenida con contenidos en menús horizontal y laterales. http://www.bibliored30.com (18.09.2015).

Bibliored3.0 (2015b). Transmedia, crossmedia, multiplataforma: fuentes de información (selección) http://www.bibliored30.com/search/?q=transmedia (Consulta: 18.09.2015). 
Biblogsfera (2014) Directorio de blogs relacionados com Biblioteconomía y Documentación: http://biblogsfera.com/ http://biblogsfera.com/bibliored-3-0/ http://biblogsfera.com/tag/lopez-yepes-alfonso/ (Consulta: 18.09.2015).

Biblogtecarios (2015). Blog colaborativo de Biblioteconomía y Documentación. http://www.biblogtecarios.es/firmasinvitadas/bibliored-3-0-multimedia-sobre-ciencias-dela-informacion-comunicacion-en-bibliotecas-servicios-universitarios/ (Consulta: 18.09.2015).

Centro Cultural España La Paz (2014). Taller "Archivos audiovisuales en línea y medios de comunicación: hacia una red iberoamericana de patrimonio audiovisual" en el marco de CIBDA 2014. VI Congreso Internacional de Bibliotecología, Documentación, Archivística y Museología (La Paz-Bolivia, 13-17 octubre 2014), organizado por el Colegio de Profesionales en Ciencias de la Información de Bolivia: http://www.bibliored30.com/2014/11/bolivia-taller-archivosaudiovisuales.html http://www.anabad.org/noticias-anabad/26-general/2869-vi-congresointernacional-de-bibliotecologia-documentacionarchivistica-y-muesologia.htm (Consulta: 18.09.2015).

Cuevas-Cervero, Aurora; Marques, Márcia; Sales Paixâo, Pablo Boaventura (2014). A alfabetizaçao que necesitamos: informaçao e comunicaçao para a cidadania. Inf.\&Soc.:est, Joâo Pessoa, v.24, n.2, p.35-48.

Díaz Noci, J. y Tous Rovirosa, A. (2012). La audiencia como autor: narrativas transmedia y propiedad intelectual del público. Algunas reflexiones jurídicas. El profesional de la información, septiembre-octubre, v. 21, pp.458-467.

http://ddd.uab.cat/pub/artpub/2012/112794/proinf a2012v21n5p458.pdf $\quad$ (Consulta: 18.09.2015).

EDA (2014) Blog El documentalista audiovisual. Bibliored 3.0: Multimedia sobre ciencias de la información y comunicación en bibliotecas y servicios universitarios: http://eldocumentalistaudiovisual.com/?s=bibliored+3.0 13 (Consulta: 18.09.2015).

Fundación Telefónica (2014). Transmedia y nuevos contenidos digitales.

http://www.fundaciontelefonica.com/conferencias/transmedia-y-nuevos-contenidosdigitales/ (Consulta: 18.09.2015). Ciclo Transmedia en Youtube. Narrativa Transmedia (1 ${ }^{\underline{a}}$ sesión. Parte 1 y parte 2)

https://www.youtube.com/playlist?list=PLgMP8Zkx8JptSInIr4FD22GGyPH2BdlV\& ga=1.178758817.440859546.1438073982 (Consulta: 18.09.2015).

García Jiménez, Antonio; Valle Gastaminza, Félix del (2015). Nuevos retos de la documentación en los medios de comunicación. Index Comunicación, no 5 (3), págs. 11-15. 
http://journals.sfu.ca/indexcomunicacion/index.php/indexcomunicacion/article/view/202/1 74(Consulta: 18.09.2015).

González Fernández-Villavicencio, Nieves (2015). WebCast sobre Métricas de la web social y bibliotecas. Aprender 3C, comunidad de conocimiento colaborativa, 18 septiembre 2015. http://aprender3c.org/metricas-de-la-web-social-y-bibliotecas/ (Consulta: 18.09.2015).

Grandío, Mar (2015). Alfabetización transmedia en los jóvenes universitarios. I Jornadas "Nuevos espacios para la alfabetización e inteligencias múltiples", 7 de mayo 2015. Murcia, Facultad de Matemáticas, Universidad de Murcia, emisión televisión universidad de Murcia: https://tv.um.es/video?id=71951 (Consulta: 18.09.2015). En I Jornadas "Nuevos espacios para la alfabetización e inteligencias múltiples" http://eventos.um.es/event detail/2028/detail/ijornadas-andquot;nuevos-espacios-para-la-alfabetizacion-e-inteligenciasmultiplesandquot;:html (Consulta: 18.09.2015).

HISPANA-EUROPEANA (2015). Presencia del Servicio de Documentación de la Universidad Complutense y de sus actuaciones académico-científicas. http://www.bibliored30.com/search/search/?q=hispana (Consulta: 18.09.2015).

http://www.bibliored30.com/search/?q=europeana (Consulta: 18.09.2015).

Icono 14 (2015). Invitación a colaborar en preparación número monográfico sobre transmedia, crossmedia, multiplataforma oy otras temáticas relacionadas. http://www.icono14.net/ojs/index.php/icono14/announcement/view/11 (Consulta: 18.09.2015).

López Yepes, Alfonso (2007-2013/2015). E-prints Complutense. http://eprints.ucm.es/view/people/L=F3pez Yepes=3AAlfonso=3A=3A.html

(Consulta: 18.09.2015).

López Yepes, A. (2015). Patrimonio audiovisual en bibliotecas y servicios universitarios a propósito del servicio de documentación multimedia de la universidad complutense: propuestas de colaboración hispano-brasileña en el acceso a la información y memoria. En IV Seminario Hispano-Brasileño de Investigación en Información, Documentación y Sociedad, 24-26 junio 2015.

http://soac.unb.br/index.php/SHBPIDS/4 (Consulta: 18.09.2015).

Miranda, Antônio y Simeâo, Elmira (2014). Da comunicaçâo extensiva ao hibridismo da Animaverbivocovisualidade - AV3. Inf. \& Soc.:Est., João Pessoa, v.24, n.3, p. 49-62, set./dez. http://www.ies.ufpb.br/ojs/index.php/ies/article/view/19075/12401

(Consulta: 2015.10.02). 
Peres, Mônica; Miranda, Antônio; Simeâo, Elmira (2015). Competência em informaçâo e acervamento: produçâo coletiva e em animaverbivocovisualidade - AV3. IV Seminário de Competência em Informaçâo. Câmpus de Marilia, UNESP, 24-26 junho. http://slideplayer.com.br/slide/5582928/ (Consulta: 2015.10.02).

Ponjuán, Gloria; Pinto, María; Uribe-Tirado, Alejandro (2015). Conceptualización y perspectivas de la alfabetización informacional en Iberoamérica: un estudio Delphi. Information Research, vol. 20 no. 3, septiembre 2015. http://InformationR.net/ir/20-3/paper680.html).

http://www.informationr.net/ir/20-3/paper680.html\#.VgErUd tmko (Consulta: 18.09.2015).

Ramos Simón, Fernando y Arquero Avilés, Rosario (2015). La plataforma del patrimonio cultural europeo. Madrid, Trea, 304 págs.

REBIUN (2015). Directorio de repositorios y acceso a repositorio Universidad Complutense Madrid, E-prints Complutense. http://www.rebiun.org/repositorios/Paginas/Directorio-deRepositorios-Institucionales-REBIUN.aspx $\quad$ http://roar.eprints.org/463/ (Consulta: 18.09.2015).

Scolari, Carlos (2013). Narrativas transmedia: cuando todos los medios cuentas. Bilbao, Deusto S.A. Ediciones.

Servicio Documentación Multimedia (1993-2015). Actuaciones académicas: proyectos de investigación, publicaciones electrónicas, portales, redes, revista digital... Madrid, Dpto. Biblioteconomía y Documentación, Facultad de Ciencias de la Información, Universidad Complutense: http://www.multidoc.es (Consulta: 18.09.2015).

Servicio Documentación Multimedia (2015). Vídeo sobre contenidos y objetivos del Servicio. en menú lateral derecho en portada del sitio, realizado por José Ma Echegaray Maldonado y Adrián Gallardo Gómez, junio 2015, 4'11": https://www.youtube.com/watch?v=wuTIS6ZbHnQ\&feature=youtu.be Consulta: 18.09.2015).

Universo Abierto (2014). Blog de la biblioteca de la Facultad de Traducción y Documentación de la Universidad de Salamanca.

http://www.universoabierto.com/?s=bibliored+3.0 (Consulta: 18.09.2015).

Uribe-Tirado, Alejandro (2015). Coordinación de la Red Latinoamericana de Competencias Informacionales RedLADCI: $\quad$ http://alfincolombia.blogspot.com.es/2015/09/redlatinoamericana-de-competencias.html (Consulta: 18.09.2015). 